\title{
Vitiligo-like depigmentation after pembrolizumab treatment in patients with non-small cell lung cancer: a case report
}

\author{
Sook Jung Yun ${ }^{1}$, In-Jae Oh ${ }^{2,3}$, Cheol Kyu Park ${ }^{2,3}$, Young-Chul Kim ${ }^{2,3}$, Hyeon Bin Kim ${ }^{1}$, Hee-Kyung Kim ${ }^{3}$, \\ A. Ram Hong ${ }^{3}$, In-Young Kim ${ }^{2,4}$, Sung-Ja Ahn ${ }^{2,5}$, Kook-Joo Na ${ }^{2,6}$, Yoo-Duk Choi ${ }^{2,7}$ \\ ${ }^{1}$ Department of Dermatology, Chonnam National University Medical School, Gwangju, Korea; ${ }^{2}$ Lung and Esophageal Cancer Clinic, Chonnam \\ National University Hwasun Hospital, Jeonnam, Korea; ${ }^{3}$ Department of Internal Medicine, ${ }^{4}$ Department of Neurosurgery, ${ }^{5}$ Department of Radiation \\ Oncology, ${ }^{6}$ Department of Thoracic Surgery, ${ }^{7}$ Department of Pathology, Chonnam National University Medical School, Gwangju, Korea \\ Correspondence to: In-Jae Oh, MD, PhD. Lung and Esophageal Cancer Clinic, Chonnam National University Hwasun Hospital, Jeonnam 58128, \\ Korea. Email: droij@chonnam.ac.kr.
}

\begin{abstract}
Immune checkpoint inhibitors (ICIs) targeting programmed death-1 (PD-1) pathway have significantly improved outcomes for patients with a variety of malignancies, including non-small cell lung cancer (NSCLC). In contrast, the incidence of immune-related cutaneous adverse events such as vitiligo have been on the rise because of the increasing use of ICIs. Vitiligo-like depigmentation has been reported in only $2.0 \%$ to $8.3 \%$ of patients with melanoma and is considered a favorable prognostic factor. However, it has been rarely reported in patients with non-melanoma malignancies. We describe a case of vitiligo-like skin depigmentation after pembrolizumab use in a patient with stage IV NSCLC. Multiple ill-defined painless and non-pruritic depigmented patches appeared on the patient's hands, scrotum, and lower lip after five months of pembrolizumab. We continued treatment with pembrolizumab $2 \mathrm{mg} / \mathrm{kg}$ for 14 months with close monitoring of vitiligo lesions until the progression of brain metastasis, but the vitiligo-like depigmentation did not improve by the combined excimer laser and topical corticosteroid therapy. Clinicians should be aware that immune-related cutaneous adverse events such as vitiligo-like depigmentation are not limited to cases of melanoma but arise as a direct result of anti-PD-1 therapy.
\end{abstract}

Keywords: Immunotherapy; lung neoplasm; programmed cell death-1 receptor; vitiligo; case report

Submitted Mar 05, 2020. Accepted for publication Jun 03, 2020.

doi: $10.21037 /$ tlcr-20-386

View this article at: http://dx.doi.org/10.21037/tlcr-20-386

\section{Introduction}

The development of immune checkpoint inhibitors (ICIs) that target the cytotoxic T-lymphocyte-associated antigen 4 and programmed death-1/ligand 1 (PD-1/PD-L1) pathways has significantly improved the outcomes of patients with a variety of malignancies including non-small cell lung cancer (NSCLC) (1). The PD-1/PD-L1 interaction leads to the inactivation of cytotoxic T-cells and downregulation of the immune response, thereby promoting cancer progression and metastasis. The blocked interaction between tumor cells and activated T-cells permits the immune system to remain active (2). In the United States, Europe, and Korea, anti-PD-1 inhibitors (nivolumab and pembrolizumab) and
anti-PD-L1 inhibitors (atezolizumab) have been approved as second-line treatment for NSCLC patients with PD-L1 expression $(2,3)$.

Despite the durable response to ICIs, the immunerelated adverse events (irAEs) can affect multiple organs of the body, and are most commonly observed in the skin, gastrointestinal tract, and lungs, as well as the endocrine, musculoskeletal, renal, nervous, hematologic, cardiovascular, and ocular systems (3). Fatal adverse events are rare; however, treatment interruption or discontinuation, compromised quality of life, or morbidity may occur (3-6). Therefore, clinical practice guideline recommends there should be a high level of suspicion that new symptoms are treatment related (3). 

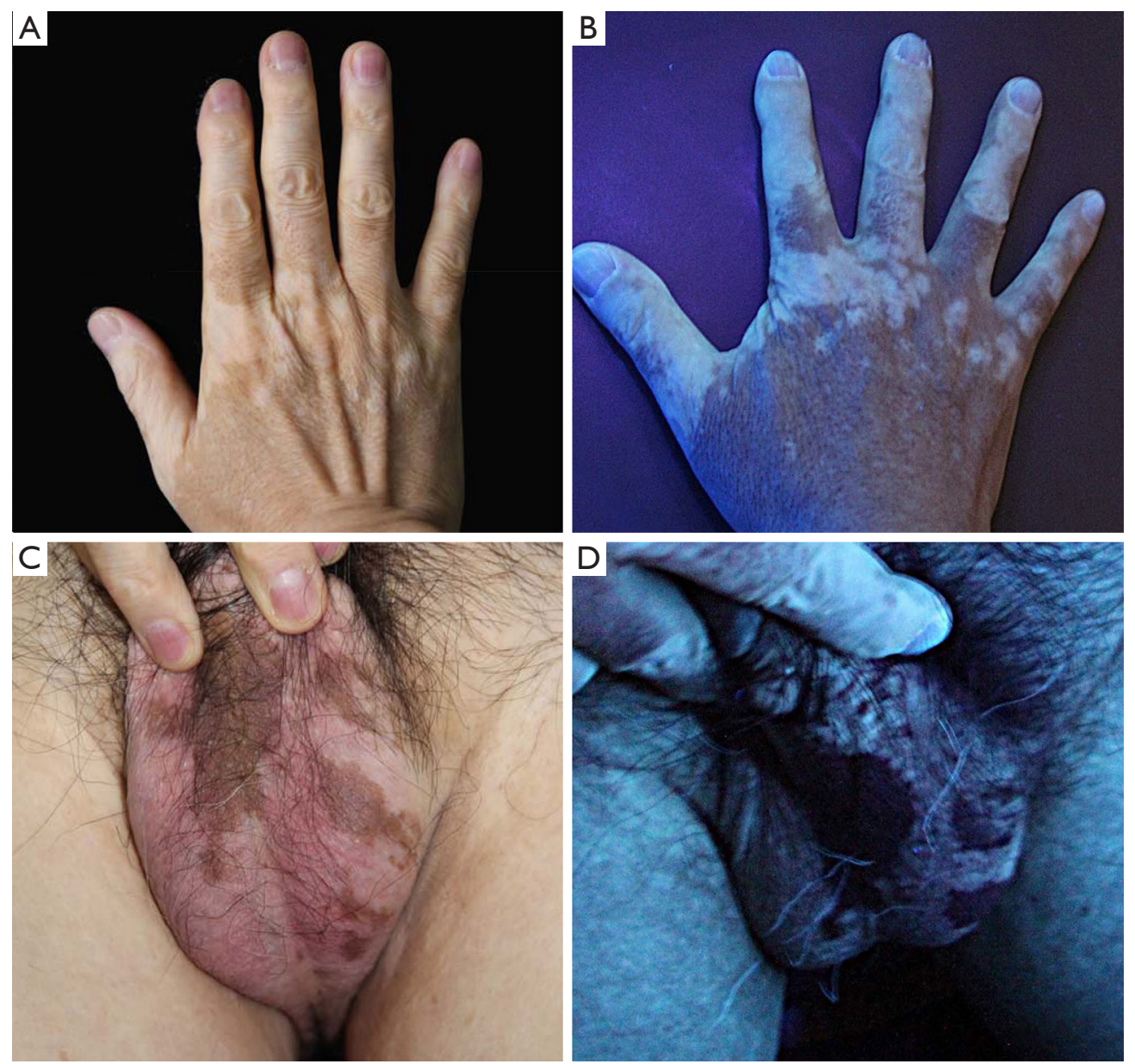

Figure 1 Dermatologic findings of vitiligo-like depigmentation. (A,C) Multiple depigmented patches on the patient's hand and scrotum. (B,D) Marked accentuation of these depigmented patches on wood lamp examination.

During anti-PD-1 monotherapy, cutaneous irAEs such as vitiligo, psoriasis, and lichenoid dermatitis can develop. But vitiligo-like depigmentation has been rarely reported in patients with non-melanoma malignancies (4). In this study, we describe a case of vitiligo-like skin depigmentation after 5 months of pembrolizumab use in a patient with NSCLC. We present the following case in accordance with the CARE Reporting checklist (available at http://dx.doi. org/10.21037/tlcr-20-386).

\section{Case presentation}

A 63-year-old Korean male patient presenting with nonpruritic, variable-sized depigmented patches on his hands and genitalia was presented at the Chonnam National University Hwasun Hospital on August 29, 2018 (Figure 1).
He was a 30 pack-year ex-smoker since 2005. He had undergone a left upper lobe lobectomy with mediastinal lymph node dissection on November 10, 2015 and was diagnosed with papillary adenocarcinoma (pT2aN2M0, stage IIIA). The adenocarcinoma recurred in supraclavicular lymph nodes and the cerebellum after 5 and 11 months after completion of adjuvant chemoradiotherapy, respectively (Figure 2). He received palliative radiotherapy for the supraclavicular lymph nodes, and gamma knife radiosurgery for cerebellar metastasis.

Based on molecular analysis using surgical tissue, there was epidermal growth factor receptor exon 20 insertion mutation by real-time polymerase chain reaction without anaplastic lymphoma kinase rearrangement in break-apart fluorescence in situ hybridization. Immunohistochemistry (IHC) using PD-L1 IHC 22C3 pharmDx (Agilent 

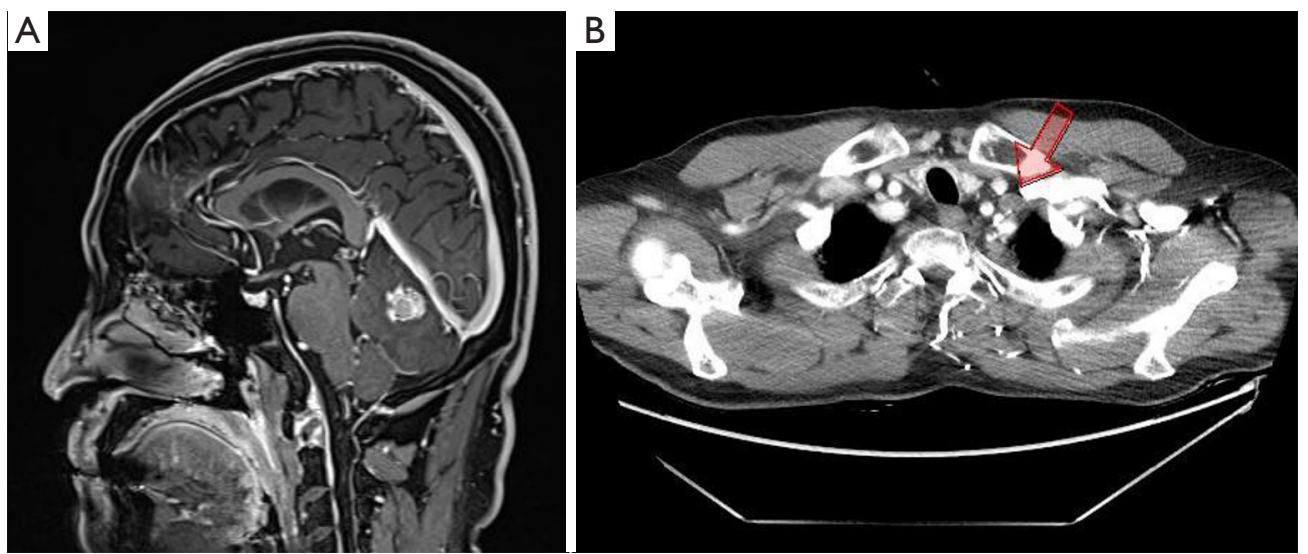

Figure 2 Radiologic findings of recurrences. (A) Magnetic resonance image of the brain showing a $1.6 \mathrm{~cm}$ heterogeneous mass in the cerebellar vermis with perilesional edema. (B) Computed tomography scan showing a slightly enlarged lymph node in left supraclavicular area (shown by the arrow).

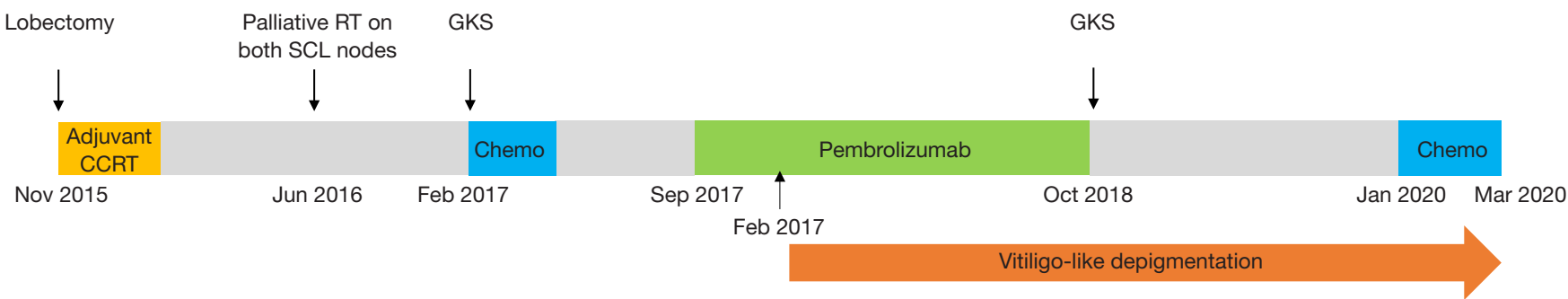

Figure 3 Time line and duration of each treatment. Vitiligo-like depigmentation appeared 5 months after pembrolizumab use, and has been maintained for 25 months until now. Gray color indicates periods without cancer treatment. CCRT, concurrent chemoradiation; RT, radiotherapy; SCL, supraclavicular; GKS, gamma knife surgery.

Technologies, Santa Clara, CA, USA) showed high PD-L1 expression with tumor proportion score $70 \%$.

Because the patient could not tolerate the 2 nd cycle of chemotherapy with pemetrexed $500 \mathrm{mg} / \mathrm{kg}$ and cisplatin $60 \mathrm{mg} / \mathrm{kg}$, pembrolizumab $2 \mathrm{mg} / \mathrm{kg}$ was administered from September 6, 2017 (Figure 3). Additionally, $0.1 \mathrm{mg}$ synthroid (levothyroxine) was administered from November 8, 2017 to control immune-related hypothyroidism. A thyroid function test revealed the following: triiodothyronine (T3): $0.779 \mathrm{ng} / \mathrm{mL}$, free thyroxine (T4): $0.526 \mathrm{ng} / \mathrm{dL}$, and thyroid-stimulating hormone (TSH): $70.79 \mathrm{IU} / \mathrm{mL}$; there was no evidence of autoantibodies, including antimicrosomal and antithyroglobulin antibodies.

Five months after the initiation of pembrolizumab, multiple ill-defined painless and non-pruritic depigmented patches appeared on the patient's hands, scrotum, and lower lip, which were markedly accentuated on Wood lamp examination. The patient no longer presented with fever, arthralgia, fatigue, or any indication of connective tissue disease. There was no history of dermatosis before pembrolizumab use. Food- and travel-related issues, as well as trauma was ruled out as being potential causes. The skin biopsy was unremarkable in appearance, and the absence of melanocytes in the epidermis was confirmed by Melan-A immunohistochemical staining (Figure 4). The application of a topical corticosteroid $45 \mathrm{~g}$ and biweekly excimer laser therapy resulted in no significant improvement.

The patient continued using pembrolizumab for 14 months with stable disease. However, administration of the drug was discontinued because of the development of brain metastasis, which required gamma knife radiosurgery. The asymptomatic vitiligo-like lesions persisted during the pembrolizumab use and remained persistent for 25 months. Written informed consent (IRB approved number: CNUHH 2020-039) was obtained from the patient for the publication of this case report and any 

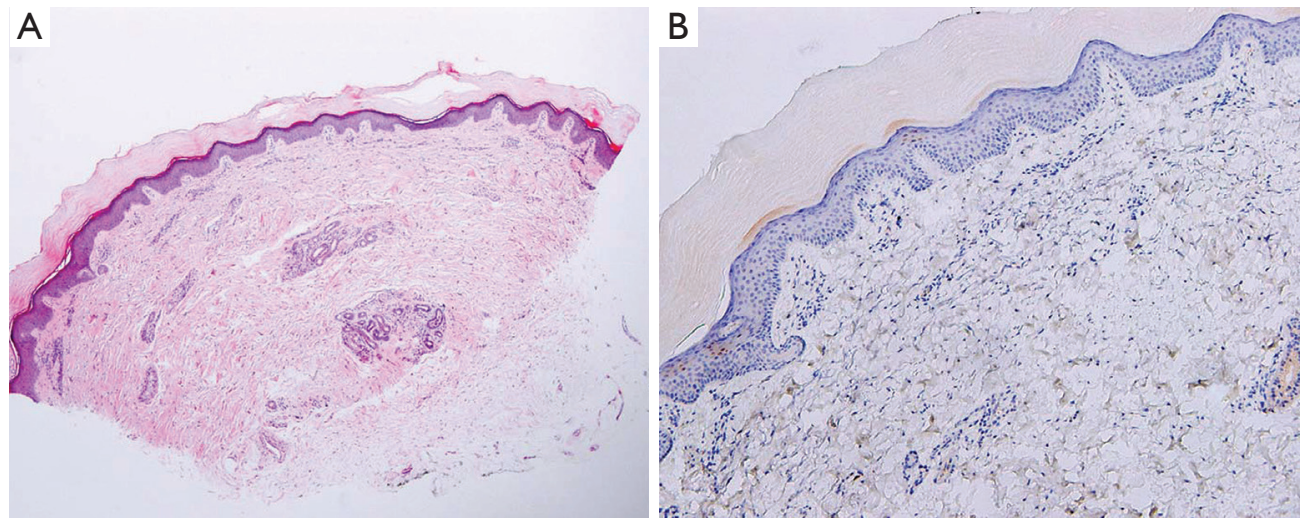

Figure 4 Histopathologic findings. (A) A punch biopsy from the hand dorsum showing unremarkable skin (HE, $\times 40)$. (B) There were no melanocytes in the epidermis per Melan-A immunohistochemical staining $(\times 100)$.

accompanying images.

\section{Discussion}

Vitiligo-like depigmentation has been reported in only $2.0 \%$ to $8.3 \%$ of patients with melanoma (1). Additional studies have reported the rarity of this occurrence, especially in patients with non-melanoma metastatic cancers (7-10). Nivolumab-induced vitiligo-like depigmentation in NSCLC has been reported in various studies $(7,8,10-13)$. However, vitiligo-like depigmentation resulting from the use of pembrolizumab in NSCLC has not been reported.

Vitiligo is defined as the appearance of hypopigmented areas on the skin, and is classified as either localized or generalized according to the distribution of the lesions (14). This complex phenomenon is caused by the destruction of melanocytes, and is associated with numerous genetic and autoimmune conditions (8). In malignant melanoma, it is assumed that the release of melanoma-associated antigens shared by normal melanocytes results from the destruction of melanoma cells; the appearance of vitiligo is therefore an indication of immune activation against the melanocyte antigen, and is an independent favorable prognostic factor $(7,8,14)$. Kosche et al. postulated that a common antigen exists among NSCLCs, melanomas, and healthy melanocytes, which are targeted by activated T-cells (8). Labeling immunotherapy-associated vitiligo as a favorable prognostic factor in NSCLC management is yet to be confirmed.

In this study, we described a case of vitiligo-like depigmentation after 5 months of pembrolizumab $2 \mathrm{mg} / \mathrm{kg}$ use in stage IV NSCLC with brain metastasis. Although the patient had gone through quite a complicated intervention history such as surgery, adjuvant chemoradiotherapy, palliative radiotherapy, gamma knife surgery and levothyroxine medication, there was no specific history of dermatosis before pembrolizumab use. So we could judge that the depigmentation was caused by pembrolizumab. The skin lesions persisted without exacerbation or improvement for 9 months. There are two interesting points in this case. First, we do could learn some message regarding whether it's a prognostic factor for patients with NSCLC. While the depigmentation had been existing, pembrolizumab was discontinued at 14 months due to the progression of brain metastasis. Therefore, anti-PD1 inhibitor-associated vitiligo in NSCLC may not be a favorable factor unlike melanoma. Second point is that the skin lesions persisted even after pembrolizumab was finished. This suggests that anti-PD1 immunotherapy may cause irreversible skin manifestation. However, our case alone has limitation in determining the cancer prognosis and reversibility of vitiligo-like depigmentation.

Because of the increasing use of ICIs, the incidence of immune-related cutaneous adverse events (ircAEs) has been on the rise. The severity of irAEs ranges from grade 1 (pruritus or maculopapular rash) to grade 4 (StevensJohnson syndrome); severe ircAEs (grade $\geq 3$ ) occur in approximately $2 \%$ to $10 \%$ of the treated patients (1). The majority of ircAEs are classified as grade 1. Asymptomatic vitiligo is commonly observed without treatment; however, there was a case of nivolumab-induced vitiligo successfully treated with narrowband ultraviolet B phototherapy (15). In another study, compared to excimer laser monotherapy, $308 \mathrm{~nm}$ excimer laser therapy combined with topical drugs 
showed a superior response (16). In our study, we continued with pembrolizumab for 14 months, but the vitiligo-like depigmentation did not improve with combined excimer laser and topical corticosteroid therapy.

\section{Conclusions}

The detailed nature of non-melanoma malignancies, including NSCLC, is not fully understood. However, clinicians should be aware that ircAEs such as vitiligo-like depigmentation are not limited to cases of melanoma, but rather arise as a direct result of anti-PD-1 therapy. And immunotherapy could be continued for mild ircAE case with close monitoring.

\section{Acknowledgments}

We would like to thank Editage (www.editage.com) for their assistance with English language editing.

Funding: None.

\section{Footnote}

Peer Review File: Available at http://dx.doi.org/10.21037/ tlcr-20-386

Conflicts of Interest: All authors have completed the ICMJE uniform disclosure form (available at http://dx.doi. org/10.21037/tlcr-20-386). The authors have no conflicts of interest to declare.

Ethical Statement: The authors are accountable for all aspects of the work in ensuring that questions related to the accuracy or integrity of any part of the work are appropriately investigated and resolved. All procedures performed in studies involving human participants were in accordance with the Declaration of Helsinki (as revised in 2013). Written informed consent (IRB approved number: CNUHH 2020-039) was obtained from the patient for the publication of this case report and any accompanying images.

Open Access Statement: This is an Open Access article distributed in accordance with the Creative Commons Attribution-NonCommercial-NoDerivs 4.0 International License (CC BY-NC-ND 4.0), which permits the noncommercial replication and distribution of the article with the strict proviso that no changes or edits are made and the original work is properly cited (including links to both the formal publication through the relevant DOI and the license). See: https://creativecommons.org/licenses/by-nc-nd/4.0/.

\section{References}

1. Phillips GS, Wu J, Hellmann MD, et al. Treatment Outcomes of Immune-Related Cutaneous Adverse Events. J Clin Oncol 2019;37:2746-58.

2. Park HY, Oh IJ, Kho BG, et al. Clinical Characteristics of Korean Patients with Lung Cancer Who Have Programmed Death-Ligand 1 Expression. Tuberc Respir Dis (Seoul) 2019;82:227-33.

3. Brahmer JR, Lacchetti C, Schneider BJ, et al. Management of Immune-Related Adverse Events in Patients Treated With Immune Checkpoint Inhibitor Therapy: American Society of Clinical Oncology Clinical Practice Guideline. J Clin Oncol 2018;36:1714-68.

4. Sanlorenzo M, Vujic I, Daud A, et al. Pembrolizumab Cutaneous Adverse Events and Their Association With Disease Progression. JAMA Dermatol 2015;151:1206-12.

5. Kim JS, Nam TS, Kim J, et al. Myasthenia gravis and myopathy after nivolumab treatment for non-small cell lung carcinoma: A case report. Thorac Cancer 2019;10:2045-9.

6. Heinzerling L, Goldinger SM. A review of serious adverse effects under treatment with checkpoint inhibitors. Curr Opin Oncol 2017;29:136-44.

7. Liu RC, Consuegra G, Chou S, et al. Vitiligo-like depigmentation in oncology patients treated with immunotherapies for nonmelanoma metastatic cancers. Clin Exp Dermatol 2019;44:643-6.

8. Kosche C, Mohindra N, Choi JN. Vitiligo in a patient undergoing nivolumab treatment for non-small cell lung cancer. JAAD Case Rep 2018;4:1042-4.

9. Dumbrava EI, Ivan D, Subbiah V. Hypopigmented Skin Lesions After Immunotherapy. JAMA Oncol 2018;4:1118-9.

10. Zhao ZM, Liu SC, Xu XJ, et al. Treatment of Skin Reaction Induced by Nivolumab Combined with Radiotherapy in Non-small Cell Lung Cancer: A Case Report. Chin Med Sci J 2018;33:183-7.

11. Nishino K, Ohe S, Kitamura M, et al. Nivolumab induced vitiligo-like lesions in a patient with metastatic squamous cell carcinoma of the lung. J Thorac Dis 2018;10:E481-4.

12. Zarogoulidis $P$, Huang $H$, Tsiouda T, et al. Immunotherapy "Shock" with vitiligo due to nivolumab administration as third line therapy in lung adenocarcinoma. Respir Med 
Case Rep 2017;22:283-6.

13. Uenami T, Hosono $Y$, Ishijima M, et al. Vitiligo in a patient with lung adenocarcinoma treated with nivolumab: A case report. Lung Cancer 2017;109:42-4.

14. Hua C, Boussemart L, Mateus C, et al. Association of Vitiligo With Tumor Response in Patients With Metastatic Melanoma Treated With Pembrolizumab. JAMA Dermatol 2016;152:45-51.

Cite this article as: Yun SJ, Oh IJ, Park CK, Kim YC, Kim HB, Kim HK, Hong AR, Kim IY, Ahn SJ, Na KJ, Choi YD. Vitiligo-like depigmentation after pembrolizumab treatment in patients with non-small cell lung cancer: a case report. Transl Lung Cancer Res 2020;9(4):1585-1590. doi: 10.21037/tlcr-20-386
15. Miyagawa T, Kadono T, Masui Y, et al. Nivolumabinduced vitiligo successfully treated with narrowband UVB phototherapy. Eur J Dermatol 2017;27:656-8.

16. Bae JM, Hong BY, Lee JH, et al. The efficacy of 308-nm excimer laser/light (EL) and topical agent combination therapy versus EL monotherapy for vitiligo: A systematic review and meta-analysis of randomized controlled trials (RCTs). J Am Acad Dermatol 2016;74:907-15. 